\title{
A Comparative Study of Biological Evolution and Cultural Evolution from the Perspective of Evolution
}

\author{
Shuyan Zhao \\ Tiangong University, Tianjin, China \\ Email: zhaoshuyan5@126.com
}

How to cite this paper: Zhao, S.Y. (2021) A Comparative Study of Biological Evolution and Cultural Evolution from the Perspective of Evolution. Open Access Library Journal, 8: e7673.

https://doi.org/10.4236/oalib.1107673

Received: June 21, 2021

Accepted: July 13, 2021

Published: July 16, 2021

Copyright $\odot 2021$ by author(s) and Open Access Library Inc.

This work is licensed under the Creative Commons Attribution International License (CC BY 4.0).

http://creativecommons.org/licenses/by/4.0/

\section{(c) (i) Open Access}

\begin{abstract}
Language is the key feature of distinguishing human from other animals. The history of language evolution can be traced back to ancient Egypt. Since Darwin published The Origin of Species for more than a century, the tradition of studying language with the idea of evolution has been very popular. It is found that the research on language evolution is an interdisciplinary study, and its progress involves the latest development of many disciplines. In order to reach a consistent view on language evolution, more assumptions and discussions are needed. This paper reviews the origin of evolutionism language view, focuses on two viewpoints of language origin research under the framework of evolutionism: Gradualism and Mutation Theory. It also makes a simple comparative analysis of biological evolution and cultural evolution, and discusses the concept of language evolution from the theoretical basis and evolution mechanism.
\end{abstract}

\section{Subject Areas}

Linguistics

\section{Keywords}

Language Evolution, The Origin of Language, Language Gene, Language Memes

\section{Introduction}

The origin of language has always been a point of concern in linguistics. Where does human language come from? What is the oldest language? When did the language come into being? Why only humans have language? There has been no 
breakthrough in the research on the origin of language for a long time. The main reason is the difficulty of obtaining evidence. Scholars have been working hard to find answers to these questions, including not only linguistics, but also philosophy, science, theology, biology, and many other fields.

Language evolution has gone through a long process of two million years, but humans have only explored the laws of language evolution and development for 150 years. Foreign language evolution studies have gradually reached consensus, and substantial research results have been achieved. However, although Chinese linguistics is a long-standing study, most of the documents of more than 2000 years discuss Chinese characters, phonology, and grammar. There is little focus on other languages; in exploring the origin and evolution of the Chinese language, apart from a few famous quotes by Xunzi and other sporadic theories, there is hardly any systematic research [1].

In the process of language evolution research, many scholars analyze language evolution from different research fields, such as Theology, Medicine, Psychology, and Biology. However, this article tries to review the proposal of language evolution briefly, conduct a simple comparative analysis of biological evolution and cultural evolution, and try to better understand the current issues of language origin and evolution from different theoretical levels.

\section{A Proposal of Language Evolution}

Darwin published The Origin of Species in 1859 [2]. The core ideas are: 1) If we trace the roots, animals and plants originated from a few common ancestors. The offspring of a species will undergo a gradual change. The offspring of a change is a new variant. There is no essential boundary between a variant and a new species, because a variant can also form a new species. 2) Natural selection plays a central role in species evolution. 3) The existence of transitional species. Darwin's theory of evolution emphasizes the core role of natural selection. The object "captured" by natural selection is beneficial changes in species. Such changes are subtle, gradual, and continuous. Therefore, transitional species must have existed [3].

German linguist August Scheilurer (1821-1868) under the influence of his colleague Ernst Haeckel (E. Haeckel, 1834-1919) at Friedrich-Schiller-Universität Jena began to use biology evolutionary model to study language development. Schleicher believes that "language is a natural organism. They are formed without the determination of human will, and grow, develop, and die according to certain laws" [4]. Schleicher associates linguistics with Botany, and believes that the genus spoken by natural researchers, linguists call it a language family; for several closely related genera, linguists also call a language family as language of relatives. Several species of a genus in biology are called language family in our linguistics. A dialect or local dialect of a language is a number of subspecies of a species. The smaller dialect is equivalent to variation [5]. Schleicher, under the influence of Darwin, pioneered the introduction of tree diagrams depicting bio- 
logical evolutionary categories in biology into the field of language research, depicting the corresponding language pedigree tree. He compared the entire Indo-European language family to a tree, vividly describing the relationship between languages during the evolution of languages. Schleicher's genealogical tree theory puts forward the problem of genealogical classification of kinship language for the first time. In addition, he is also the first theorist to show that the branches on the tree diagram may be used to represent the time of language disappearance, that is, to reveal the occurrence, development and evolution of language through the tree diagram. Schleicher applied the theory of evolution to the study of linguistics and put forward the subject of language evolution. Since then, researchers have really started the study of language evolution.

\section{Biological Evolution: The Proposal of the Biological Faculty of Language}

Chomsky believes that Darwin's theory of natural selection evolution is difficult to explain the following problem: all human biological faculties can be seen in other organisms, but only humans have language. This seems to be a huge leap in biology, but it violates Linnaeus and Darwin's principle-Nature never leaps over [6].

Chomsky put forward language innate theory on the basis of Descartes's "innate concept". Chomsky believes that when people understand and create discourse, what people mainly rely on is not experience, but the innate language faculty. Language faculty is unique to humans, and it is like the visual system. Like the immune system, it is a "language organ" [7]. That is to say, there is a language acquisition device (LAD) in the human brain, which is embodied as Universal Grammar, which includes the common features of all human languages. Therefore, Chomsky believes that the universal feature of language structure is the innate concept and principle.

Chomsky believes that human language comes from genetic mutations rather than gradual changes. Generative linguistics represented by Chomsky believes that human language must have biological properties: the language mechanism is determined by genetics, just like other organs in the body, and can grow, develop and mature in a suitable environment. Language faculty refers to the ability of the human brain to realize language ability and specialize in language acquisition and language use. The research on the biological mechanism of language began in 1950. Handbook of Biolinguistics published by Clarence and Chomsky. For the first time, the book defines the combination of biological research results and linguistics as biolinguistics.

In the 21st century, the study of the biological mechanism of language began to combine structural genomics and the comparative study of language evolution. Lai confirmed that mutations in the FOXP2 gene can affect the development of language and speech abilities in normal brain circuits, and this gene was named the language gene. The discovery of language genes shocked the linguis- 
tics community, and undisputed scientific facts proved that human language ability is related to the complex genome and has the basis of genetic variation [8].

The essence of the study of language evolution from the perspective of biolinguistics is the study of language mechanism. Therefore, the study of the biological mechanism of language provides theoretical support for the physiological significance of language evolution, and the discussion of the biological mechanism of language further promotes the pace of the study of language evolution, and at the same time further verifies the universal applicability of Darwinian evolution theory in the field of linguistics [9].

\section{Cultural Evolution: The Presentation of Language Memes}

Meme is a concept proposed by Richard Dawkins in the book The Selfish Gene. Dawkins hopes that the term meme can describe the evolution of cultural phenomena similar to gene, and defines meme as "a unit of cultural transmission or a unit of imitation". Dawkins pointed out that various cultural phenomena achieve cultural transmission through the self-replication of memes among people [10]. The cultural unit or information unit whose information is copied by imitation is called meme. After that, many scholars, including Susan Blackmore, Richard Brodie, Aaron Lynch and so on, actively expounded the meaning and law of meme, and tried to establish the memetic theory of cultural evolution. Since the emergence of Memetics, many scholars have used Memetics to explain many phenomena in the field of social culture. In China, He Ziran introduced Memetics and promoted the application of Memetics in domestic linguistics circles.

Memetics is a new theory that explains the laws of cultural evolution based on Darwin's theory of evolution. Memetics borrows the model of biological evolution to explore the replication, spread, and evolution of memes, and tries to integrate the role of cultural inheritance into a more standard biological model of evolution. Memetics assumes that cultural elements are composed of memes, and the essence of cultural change is the constant and continuous changes of memes.

Meme, as a cultural gene, survives by copying and spreading, and language is one of its carriers. Meme is conducive to the development of language, and meme itself is copied and spread by language. It can be seen that meme is closely related to language. From the perspective of Memetics, language memes reveal the law of discourse spreading and language spreading. Furthermore, language itself is meme, which can be expressed at the level of words, sentences and even texts. It can be said that the existence of language provides the premise for the replication and dissemination of language memes, and the role of memes accelerates the evolution and variation of language.

There are many phenomena in language use that can be explained by Memetics, and the memes in language are language memes. Language Memetics reveals the laws of language development and provides a new perspective for exploring 
the evolution of language. The linguistic memes spread through imitation embody the laws of cultural evolution [11].

The "cultural evolution view" group regards memes as a bridge connecting biological evolution and cultural evolution, and believes that memes have both biological foundations and cultural appearances. They emphasized that culture and biological evolution are similar, and both are systems that can evolve. Memes with biological characteristics can spread, indicating that biological energy has an impact on cultural evolution, and also that it can change the adaptability of species.

\section{Similarities and Differences}

\subsection{Theoretical Basis}

Darwin's theory of evolution emphasizes the core role of natural selection. The object "captured" by natural selection is beneficial changes in species, which are subtle, gradual, and continuous. The theory of evolution believes that all biological species undergo an evolutionary process from low to high. The evolutionary mechanism is "natural selection and survival of the fittest". Natural selection is the driving force of biological evolution. Biological evolution and cultural evolution are both based on the theory of evolution. They believe that natural selection is the driving force of evolution, and gradually formed their own different research systems.

The theory of biological evolution accepts the basic ideas of the theory of evolution, but does not approve of the role of natural selection, and believes that the occurrence of language is the result of mutation rather than the result of a gradual process, that is, Gene Mutation Theory. The theory of biological evolution emphasizes the importance of Gene Mutation Theory, and at the same time does not deny the role of natural selection [12]. That is, the origin and evolution of language are the result of the synergistic effect of genetic mutations with biological attributes and the environment.

The theory of cultural evolution inherits Darwin's theory of natural selection, that is, the "continuity" of language evolution-human natural selection (thinking, mental structure) in the evolutionary period of animals for the increasing ability of brain representation. Language and language ability have created through two evolutionary mechanisms-natural selection and memetic selection. That is, language is an adaptive product of natural selection and memetic selection [13]. Linguistic meme is a natural selection process that is reformed through continuous duplication, imitation, dissemination, selection and evolution, in the selection and integration of new information.

\subsection{Evolutionary Mechanism}

Since the evolution of human beings is based on the evolution of culture as its main characteristic, it shows different properties and ways between it and living beings. Human beings, like all living things, the decisive concept of survival is 
adaptation. Adaptation is the process of interaction between the change of the environment caused by the organism and the change of the organism caused by the environment. From the perspective of evolutionary mechanism, biological evolution is based on the evolution of species and is realized through the regular transmission of genetic material [14]. In the biological evolution model, the gene is the replication factor, and in the cultural evolution model, the meme is the replication factor.

The theory of biological evolution believes that language is completed through mergers in the process of spreading, copying, evolving, and developing. The merged computing system was born suddenly, not the result of natural selection, but caused by genetic mutations. The merger was formed by a great leap in the process of human evolution. "Merge" is to generate a hierarchical structure and perform recursive calculations. "As long as the vocabulary concept atom is provided, the merging operation can produce infinite digital hierarchical expressions infinitely and repeatedly. If these expressions can be systematically analyzed in the conceptual system interface, this provides a 'language of thought"' [6].

The theory of language cultural evolution believes that human language ability is driven by memes, and language as a meme is a system of copying and mimicking. A meme is a unit of information in the human brain that can affect the development of events and can be replicated in other people's brains, and is a cultural communication instruction. Language memes, as the dissemination carrier of culture and information units, have been continuously disseminated, copied and evolved through imitation. Language is constantly being copied, disseminated and evolved due to memes, and language is constantly mutating due to the role of memes, and is constantly developing towards languages or language variants that are needed by humans or are conducive to human needs. When language becomes an outdated meme, it will be discarded and ignored by society.

\section{Conclusions}

Language evolution is a scientific research method to study language. The rise of the study of language evolution has promoted the development of Neurology, Biology, Psychology and other disciplines.

Biological evolution and cultural evolution discuss the different nature and attributes of language from two different levels. Language or language ability is the product of synergy between genes (biological evolution) and memes (cultural evolution). Genes play a decisive role in the origin of language, and memes play a promoting role relative to language functions. It can be said that language is a bridge between genes and memes. Memetics believes that culture is the second replication factor that appeared when human ancestors used imitation skills. Genes and memes evolved together, and memes reorganized the human brain to better transmit the copied memes [15].

Language originates from gene mutation, which is the essential feature of 
language biology; when language is produced, language is continuously spread, copied and evolved through memes, which is the functional attribute of language culture. Nowadays, the development of language depends on the genetic mutations that distinguish humans from other animals and the widespread duplication and spread of language memes. Language evolution is the continuous development and evolution of the evolution of biological structure and the use and development of language memes in verbal communication. In other words, language evolution is the result of the combined effect of biological evolution and cultural evolution.

\section{Conflicts of Interest}

The author declares no conflicts of interest.

\section{References}

[1] Wang, S. (2013) Essays on Evolutionary Linguistics. The Commercial Press, Beijing.

[2] Darwin, C. (1895) On the Origin of Species. John Murray, London.

[3] Yao, A. (2019) Chomsky's Hypothesis of Language Evolution. Modern Society, No. 15, 70-72.

[4] Wang, S. (2011) The Evolution of Evolutionary Linguistics. Contemporary Linguistics, No. 1, 1-21.

[5] Wu, W. (2013) A Survey of the Study of Language Evolution. Linguistic Research, No. 1, 89-97.

[6] Berwick, R.C. and Chomsky, N. (2016) Why Only Us: Language and Evolution. The MIT Press, Cambridge, MA. https://doi.org/10.7551/mitpress/10684.001.0001

[7] Chomsky, N. (2000) New Horizons in the Study of Language and Mind. Cambridge University Press, Cambridge. https://doi.org/10.1017/CBO9780511811937

[8] Dong, Y. and Zhang, R. (2009) FOXP2 and Human Language Ability: A New Perspective in the Study of Biological Mechanism of Language. Journal of Northeastern University (Social Science), 11, 355-359.

[9] Wu, W. (2013) About Darwin's Musical Protolanguage Theory. Shandong Foreign Language Teaching, No. 4, 43-47.

[10] Dawkins, R. (1976) The Selfish Gene. Oxford University Press, New York.

[11] Zhou, Y. and Tang, D. (2008) Linguistic Meme and Cultural Evolution. Journal of Heze University, 30, 139-142.

[12] Chomsky, N. (2005) Three Factors in the Language Design. Linguistic Inquiry, 36, 1-22. https://doi.org/10.1162/0024389052993655

[13] Luo, D. (2013) Language's Genetic and Memetic View from Evolutionary Linguistics' Perspective. Journal of Chongqing Jiaotong University (Social Sciences Edition), No. 3, 116-119.

[14] Sun, A. and Peng, X. (2002) Between Biological Evolution and Human Evolution. Journal of China Three Gorges University (Humanities \& Social Sciences), 24, 23-25.

[15] Fan, L. (2016) Biological Evolution, Cultural Evolution and Language Evolution. Chinese Social Sciences Today, No. 3, 1-2. 